\title{
X-Ray Diffraction from Isolated and Strongly Aligned Gas-Phase Molecules with a Free-Electron Laser
}

Jochen Küpper, ${ }^{1,2,3,4,5,{ }^{*}}$ Stephan Stern, ${ }^{1,2}$ Lotte Holmegaard ${ }^{1,6}$ Frank Filsinger, ${ }^{4,5, a}$ Arnaud Rouzée, ${ }^{7,8}$ Artem Rudenko, ${ }^{5,9,10}$ Per Johnsson, ${ }_{11}$ Andrew V. Martin, ${ }^{1, \mathrm{~b}}$ Marcus Adolph, ${ }^{12}$ Andrew Aquila,,${ }^{1,21}$ Saša Bajt, ${ }^{21}$ Anton Barty, ${ }^{1}$ Christoph Bostedt, ${ }^{13}$ John Bozek, ${ }^{13}$ Carl Caleman, ${ }^{1,14}$ Ryan Coffee, ${ }^{13}$ Nicola Coppola, ${ }^{1}$ Tjark Delmas, ${ }^{1}$ Sascha Epp, ${ }^{5,9}$ Benjamin Erk, ${ }^{5,9, \mathrm{c}}$ Lutz Foucar, ${ }^{5,15}$ Tais Gorkhover, ${ }^{12}$ Lars Gumprecht, ${ }^{1}$ Andreas Hartmann, ${ }^{16}$ Robert Hartmann, ${ }^{16}$ Günter Hauser, ${ }^{17,18}$ Peter Holl, ${ }^{16}$ Andre Hömke, ${ }^{5,9}$ Nils Kimmel, ${ }^{17}$ Faton Krasniqi,${ }^{5,15}$ Kai-Uwe Kühnel, ${ }^{9}$ Jochen Maurer, ${ }^{6}$ Marc Messerschmidt, ${ }^{13}$ Robert Moshammer, ${ }^{9,5}$ Christian Reich, ${ }^{16}$ Benedikt Rudek, ${ }^{5,9, \mathrm{~d}}$ Robin Santra, ${ }^{1,2,3}$ Ilme Schlichting, ${ }^{15,5}$ Carlo Schmidt, ${ }^{5}$ Sebastian Schorb, ${ }^{12}$ Joachim Schulz ${ }^{1, \mathrm{e}}$ Heike Soltau, ${ }^{16}$ John C. H. Spence, ${ }^{19}$ Dmitri Starodub,${ }^{19, \mathrm{f}}$ Lothar Strüder, ${ }^{17,20, \mathrm{~g}}$ Jan Thøgersen, ${ }^{6}$ Marc J. J. Vrakking, ${ }^{7,8}$ Georg Weidenspointner, ${ }^{17,18}$ Thomas A. White, ${ }^{1}$ Cornelia Wunderer, ${ }^{21}$ Gerard Meijer, ${ }^{4, \mathrm{~h}}$ Joachim Ullrich, ${ }^{9,5, \mathrm{~d}}$ Henrik Stapelfeldt, ${ }^{6,22}$ Daniel Rolles, ${ }^{5,15,21}$ and Henry N. Chapman ${ }^{1,2,3}$

${ }^{1}$ Center for Free-Electron-Laser Science (CFEL), DESY, Notkestrasse 85, 22607 Hamburg, Germany

${ }^{2}$ Department of Physics, University of Hamburg, Luruper Chaussee 149, 22761 Hamburg, Germany

${ }^{3}$ Hamburg Center for Ultrafast Imaging, University of Hamburg, Luruper Chaussee 149, 22761 Hamburg, Germany

${ }^{4}$ Fritz Haber Institute of the MPG, Faradayweg 4-6, 14195 Berlin, Germany

${ }^{5}$ Max Planck Advanced Study Group at CFEL, Notkestrasse 85, 22607 Hamburg, Germany

${ }^{6}$ Department of Chemistry, Aarhus University, 8000 Aarhus C, Denmark

${ }^{7}$ FOM Institute AMOLF, Science Park 104, 1098 XG Amsterdam, Netherlands

${ }^{8}$ Max-Born-Institute, Max Born Strasse. $2 a, 12489$ Berlin, Germany

${ }^{9}$ Max Planck Institute for Nuclear Physics, 69117 Heidelberg, Germany

${ }^{10}$ J. R. Macdonald Laboratory, Department of Physics, Kansas State University, Manhattan, Kansas 66506, USA

${ }^{11}$ Department of Physics, Lund University, P. O. Box 118, 22100 Lund, Sweden

${ }^{12}$ Technical University of Berlin, 10623 Berlin, Germany

${ }^{13}$ Linac Coherent Light Source, SLAC National Accelerator Laboratory, 2575 Sand Hill Road, Menlo Park, California 94025, USA

${ }^{14}$ Uppsala University, Department of Physics and Astronomy, Box 516, 75120 Uppsala, Sweden

${ }^{15}$ Max Planck Institute for Medical Research, 69120 Heidelberg, Germany

${ }^{16}$ PNSensor GmbH, 81739 Munich, Germany

${ }^{17}$ Max Planck Semiconductor Laboratory, 81739 Munich, Germany

${ }^{18}$ Max Planck Institute for Extraterrestrial Physics, 85741 Garching, Germany

${ }^{19}$ Department of Physics, Arizona State University, Tempe, Arizona 85287, USA

${ }^{20}$ University of Siegen, Emmy-Noether Campus, Walter Flex Str. 3, 57068 Siegen, Germany

${ }^{21}$ Deutsches Elektronen-Synchrotron (DESY), 22607 Hamburg, Germany

${ }^{22}$ Interdisciplinary Nanoscience Center (iNANO), Aarhus University, 8000 Aarhus C, Denmark

(Received 16 July 2013; published 28 February 2014)

\begin{abstract}
We report experimental results on x-ray diffraction of quantum-state-selected and strongly aligned ensembles of the prototypical asymmetric rotor molecule 2,5-diiodobenzonitrile using the Linac Coherent Light Source. The experiments demonstrate first steps toward a new approach to diffractive imaging of distinct structures of individual, isolated gas-phase molecules. We confirm several key ingredients of single molecule diffraction experiments: the abilities to detect and count individual scattered x-ray photons in single shot diffraction data, to deliver state-selected, e.g., structural-isomer-selected, ensembles of molecules to the x-ray interaction volume, and to strongly align the scattering molecules. Our approach, using ultrashort $\mathrm{x}$-ray pulses, is suitable to study ultrafast dynamics of isolated molecules.
\end{abstract}

X-ray free-electron lasers (XFELs) hold the promise for determining atomically resolved structures and for tracing structural dynamics of individual molecules and nanoparticles [1]. Over the last decade, ground-breaking experiments were performed at the Free-Electron Laser in Hamburg (FLASH) at DESY [2-5] and the Linac Coherent Light Source (LCLS) at the SLAC National
Accelerator Laboratory [6-12]. These experiments already begin to provide new insights into fundamental aspects of matter, such as hitherto unobserved structures of noncrystallizable mesoscopic objects [13-15] or the radiation damage induced by the short and very strong x-ray pulses $[8,16]$. However, the path to actual determination of atomically resolved structures and dynamics of single 
molecules is still long [13]. Nevertheless, related experiments on the investigation of small-molecule structures and their dynamics utilizing molecular ensembles are within reach $[13,17]$.

To be able to record structural changes during ultrafast molecular processes under well-defined conditions, it was proposed [13,17] to spatially separate shapes [18], sizes [19], or individual isomers [20-22] of complex small molecules before delivery to the interaction point of an XFEL. The molecules should be one- or three-dimensionally aligned or oriented in space $[11,12,17,23-28]$. This controlled-delivery approach would allow for the averaging of many identical patterns, similar to recent electron diffraction experiments on aligned $\mathrm{CF}_{3} \mathrm{I}$ [28] or to photoelectron imaging of 1-Ethynyl-4-fluorobenzene [12]. A controlled variation of the alignment direction in space allows one to tomographically build up the complete three-dimensional diffraction volume of individual isomers. This ensembleand pulse-averaging approach would allow working at appropriately low fluences to circumvent detrimental electronic damage processes that have been predicted [29-31] for the very high $\mathrm{x}$-ray fluences necessary to obtain classifiable single-molecule diffraction patterns. The forthcoming European XFEL facility will give the opportunity to collect patterns at a rate of 27000 per second, which could be sufficient to collect the necessary $10^{5}-10^{8}$ patterns within minutes or hours [13].

Here, we record $\mathrm{x}$-ray-diffraction patterns of ensembles of identical, state-selected, and strongly aligned 2,5-diiodo-benzonitrile (DIBN, Fig. 1) molecules in the gas phase, demonstrating the applicability of this

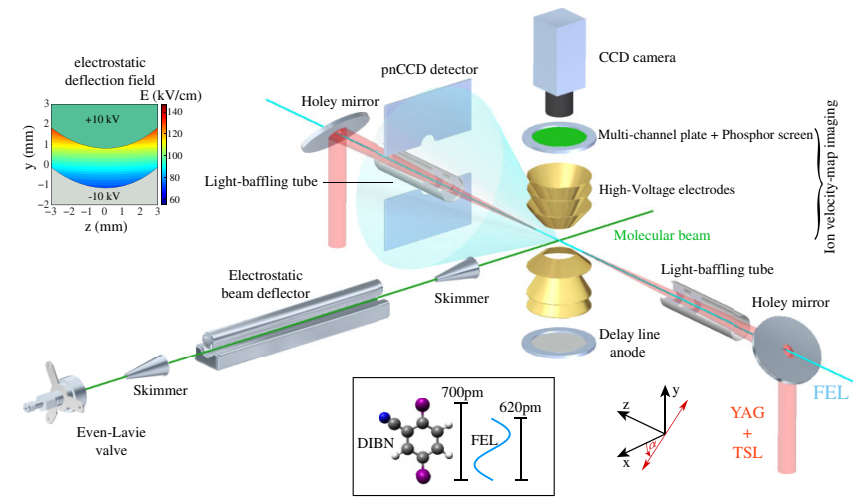

FIG. 1 (color online). Schematic view of the experimental setup: from the left, a supersonic beam with quantum-state selected molecules is delivered to the interaction point. In the center of a dual velocity map imaging spectrometer, the molecular beam is crossed by laser beams copropagating from right to left. The direct laser beams go through a gap in the pnCCD detectors that are used to record the diffraction pattern. The upper pnCCD panel is further away from the beam axis than the bottom panel in order to cover a wider range of scattering angles. In the inset, the molecular structure of 2,5-diiodobenzonitrile is depicted, together with a scale of its size, i.e., the iodine-iodine distance, and the wavelength of the $\mathrm{x}$ rays. controlled-delivery approach. Using $2 \mathrm{keV}(620 \mathrm{pm})$ radiation from the LCLS, we succeeded in observing the twocenter interference between the two iodine scattering centers, separated by approximately $700 \mathrm{pm}$, in the continuous coherent diffraction pattern. The strongly aligned samples [32] allow us to simply average the continuous diffraction patterns from a very large number of isolated molecules $[13,17]$. We restricted the angular control to one-dimensional alignment of the axis containing the two iodine atoms, as this was the solely required control for this experiment. The extension to three-dimensional alignment and orientation is straightforward for the cold, state-selected samples employed [33-35]. Moreover, we have previously demonstrated that, for more complex molecules, we could also exploit the current setup to spatially separate structural isomers and sizes [19-21].

The experiment was performed at the AMO beamline at LCLS [6,7] using the CAMP endstation [36,37] extended by a state-of-the-art molecular beam setup [38]. Figure 1 shows a scheme of the experimental arrangement. The setup contains multiple devices to simultaneously detect photons, electrons, and ions [36]. A pulsed cold molecular beam is formed by expanding a few mbar of DIBN in 50 bar of helium into a vacuum through an Even-Lavie valve [39]. The molecular beam travels through an electrostatic deflector, which disperses the molecules according to their rotational quantum states, into the target region. There, it is crossed by three pulsed laser beams: One laser beam consisting of $12 \mathrm{~ns}$ (FWHM) pulses from a Nd:YAG laser (YAG, $\lambda=1064 \mathrm{~nm}, E_{I}=200 \mathrm{~mJ}, \omega_{0}=63 \mu \mathrm{m}$, $I_{0} \approx 2.5 \times 10^{11} \mathrm{~W} / \mathrm{cm}^{2}$ ) is used to align the molecules. A second laser beam consists of $60 \mathrm{fs}$ (FWHM) pulses from a Ti:sapphire laser (TSL, $800 \mathrm{~nm}, E_{I}=400 \mu \mathrm{J}$, $\omega_{0}=40 \mu \mathrm{m}, I_{0} \approx 2.5 \times 10^{14} \mathrm{~W} / \mathrm{cm}^{2}$ ) and is used to optimize the molecular beam and the alignment without LCLS. The third beam consists of the 100 fs x-ray pulses (LCLS, $\lambda=620 \mathrm{pm}(2 \mathrm{keV}), E_{I}=4 \mathrm{~mJ}, \omega=30 \mu \mathrm{m}$, $I_{0} \approx 2 \times 10^{15} \mathrm{~W} / \mathrm{cm}^{2}$ ); we estimate that $35 \%$ of the generated $1.25 \times 10^{13} \mathrm{x}$-ray photons/pulse are transported to the experiment [40]. All three laser beams are copropagating, overlapped using dichroic (1064 and $800 \mathrm{~nm}$ ) and holey [near infrared (NIR) lasers and $\mathrm{x}$ rays] mirrors before they intersect the sample and finally leave the setup through a gap in an on-axis pnCCD camera and another holey mirror to separate the laser beams again. Time-of-flight and velocity-map-imaging (VMI) spectrometers are installed perpendicular to the horizontal plane of the molecular and laser beams to investigate the ion- and electron-momentum distributions resulting from the Coulomb explosion due to absorption of one or a few x-ray photons.

We exploit Coulomb explosion imaging of DIBN induced either by strong field ionization using the TSL pulse or through one- or two-photon ionization by the x-ray pulse to analyze the alignment of the rotational-stateselected molecules along their I-I axis. The pertinent 


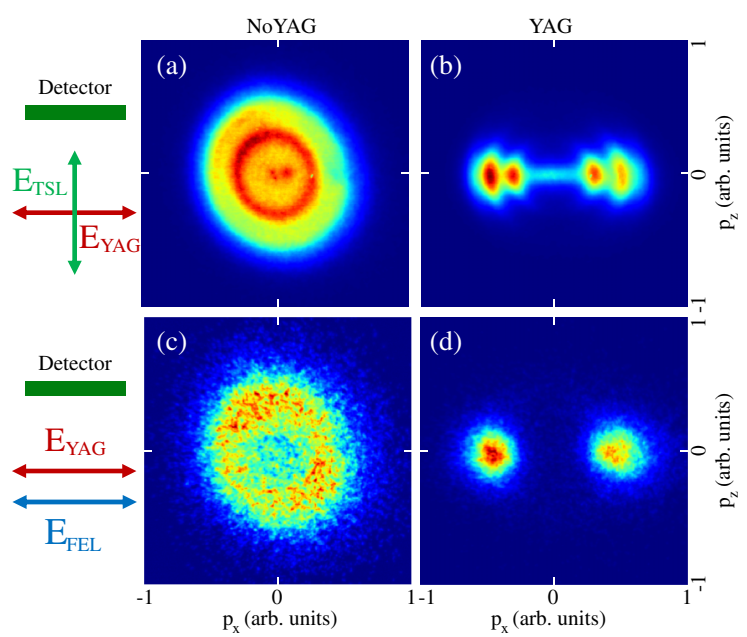

FIG. 2 (color online). $\mathrm{I}^{+}$ion images recorded with the ion-VMI detector when (a), (b) the TSL or (c), (d) the LCLS ionize and Coulomb explode the molecules. In (a) and (c), cylindrically symmetric distributions from isotropic ensembles are observed (the images are slightly distorted due to varying detector efficiencies). In (b) and (d), the horizontal alignment of the molecules, induced by the YAG, is clearly visible. In all measurements, the YAG and the LCLS are linearly polarized horizontally, parallel to the detector plane, and the TSL is linearly polarized perpendicular to the VMI detector plane.

experimental observable is the emission direction of the recoiling $\mathrm{I}^{+}$ions from the Coulomb explosion, illustrated by the $2 \mathrm{D} \mathrm{I}^{+}$ion images in Fig. 2. Without the YAG pulse, the $\mathrm{I}^{+}$images (Fig. 2) were circularly symmetric as expected for randomly aligned molecules. The circularly symmetric image obtained following ionization with the horizontally polarized LCLS beam demonstrated that the interaction of the far-off resonant radiation with the molecule was independent of the angle between the molecular axis and the x-ray polarization direction: The $\mathrm{x}$ rays were a practically unbiased ideal probe of the spatial orientation of the molecules. When the YAG pulse was included, the $\mathrm{I}^{+}$ions were strongly confined along the YAG polarization axis demonstrating tight adiabatic 1D alignment. From the corresponding 2D momentum distribution shown in Figs. 2(b) and 2(d), we extracted $\left\langle\cos ^{2} \theta_{2 \mathrm{D}}\right\rangle=$ 0.89 and $\left\langle\cos ^{2} \theta_{2 \mathrm{D}}\right\rangle=0.88$ for the TSL and LCLS ionization, respectively. This degree of alignment is in good agreement with previous measurements of adiabatic alignment of similar molecules [32] and stronger than previous alignment experiments of diatomic molecules at the LCLS [11]. This demonstrated strong alignment of complex molecules, even under the constraint conditions of a temporary setup at a FEL beamline. It was made possible by the very cold molecular beam and the adiabatic alignment conditions. The demonstrated degree of alignment fulfills the requirements for observing aligned molecule diffraction $[17,24,28]$.
In subsequent experiments we recorded the x-ray diffraction data of these aligned samples on the pnCCD cameras. For these experiments the polarization of the YAG laser was rotated clockwise by $\alpha=-60^{\circ}$. VMI data were repeatedly recorded in between diffraction experiments under the same conditions as in Fig. 2. An average value for the degree of alignment in the diffraction data of $\left\langle\cos ^{2} \theta_{2 \mathrm{D}}\right\rangle=0.84$ was derived, limited by the (changing) spatial overlap of the foci of the YAG and the LCLS beams. The obtained x-ray diffraction patterns are shown in Fig. S1 in the Supplemental Material (SM) [41]. We have analyzed diffraction data for $\approx 563000$ shots with YAG and $\approx 842000$ shots without (NOYAG), respectively, corresponding to $7 \mathrm{~h}$ (YAG) and $9 \mathrm{~h}$ (NOYAG) measurement time with LCLS operating at $60 \mathrm{~Hz}$. These data have been corrected for background and camera artifacts and individual photon hits are extracted (see SM [41]). This results in 0.20 photons/ shot, which are placed in a histogram that represents the molecular diffraction pattern (Fig. S2). By subtracting the diffraction pattern of randomly oriented molecules $\left(\mathrm{I}_{\mathrm{NOYAG}}\right)$ from the diffraction pattern of aligned molecules ( $\mathrm{I}_{\mathrm{YAG}}$ ), the background is cancelled. This includes the isotropic background originating from atomic scattering of the atoms in the DIBN molecule and the helium seed gas, as well as experimental background, e.g., scattering from apertures and rest gas.

In Fig. 3, we present these diffraction differences $\left(\mathrm{I}_{Y A G}-\mathrm{I}_{\text {NOYAG }}\right)$ for simulated [Figs. 3(a) and 3(c)] and experimentally observed [Figs. 3(b) and 3(d)] x-ray diffraction patterns. The $\mathrm{I}_{\mathrm{NOYAG}}$ data have been scaled to match the number of shots in the $\mathrm{I}_{\mathrm{YAG}}$ case. The anisotropy mainly originates in the scattering interference of the two (heavy) iodine atoms. Parts of the zeroth order scattering maximum and the first minimum (along the alignment

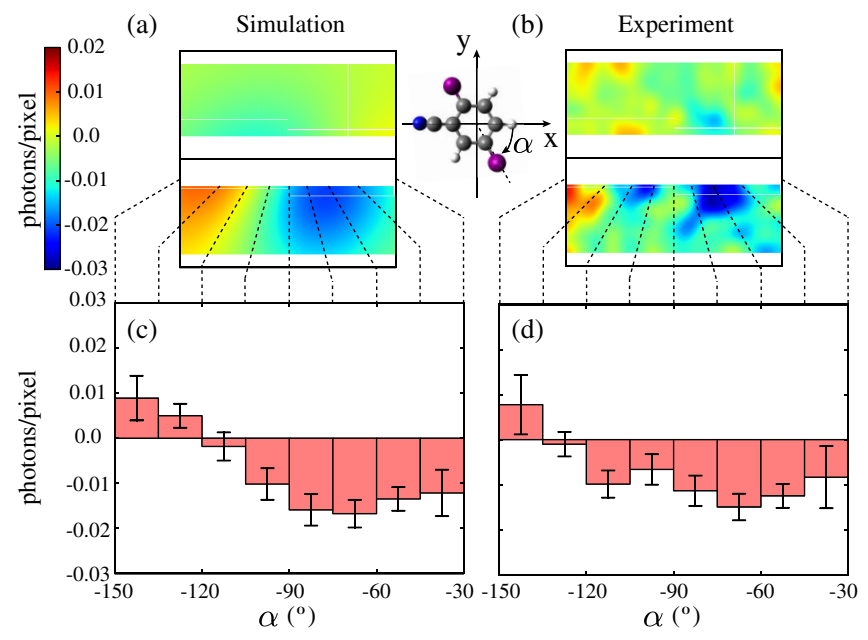

FIG. 3 (color online). Diffraction-difference $\mathrm{I}_{\mathrm{YAG}}-\mathrm{I}_{\text {NOYAG }}$ of $\mathrm{X}$-ray scattering in simulated (a) and experimental (b) X-raydiffraction patterns. Histograms of the corresponding angular distributions on the bottom pnCCD are shown in (c) and (d), respectively. Error bars correspond to $1 \sigma$ statistical errors. 
direction $\alpha=-60^{\circ}$ ) show up most prominently on the bottom pnCCD panel. The simulated $\mathrm{I}_{\mathrm{YAG}}-\mathrm{I}_{\mathrm{NOYAG}}$ image has been calculated for a molecular beam density $M$ of DIBN molecules of $M=0.8 \times 10^{8} \mathrm{~cm}^{-3}$. The error bars $\sigma$ correspond to the statistical errors from the $\mathrm{I}_{\mathrm{YAG}}-\mathrm{I}_{\mathrm{NOYAG}}$ subtraction $\left(\sigma=\sqrt{\mathrm{I}_{\mathrm{YAG}}+\mathrm{I}_{\mathrm{NOYAG}}}\right)$. The histograms in Figs. 3(c)-3(d) visualize the angular anisotropy which is well beyond the statistical error in the experimentally observed image [Fig. 3(d)], confirming the observation of x-ray diffraction from strongly aligned samples of DIBN.

To analyze which structural information can be derived from the x-ray diffraction of isolated DIBN molecules, the intensity $I(s)$ in dependence of the scattering vector $s=$ $\sin (\Theta) / \lambda$ along the alignment direction $\alpha=-60^{\circ}$ is compared to simulated models of different iodine-iodine distances. $\Theta$ is the scattering angle and $2 \Theta$ is the angle between the beam direction and a given detector point [42]. Ab initio calculations (GAMESS-US MP2/6-311 G** [43]) predict a value of $700 \mathrm{pm}$ for the iodine-iodine distance. Figure 4 shows the experimentally obtained intensity profiles $I(s)$, averaged over $-70^{\circ} \leq \alpha \leq-50^{\circ}$, together with simulated $I(s)$ profiles. Each curve is normalized to be independent of the exact molecular beam density $M$ of DIBN molecules, which merely changes the contrast, i.e., the depth of the minimum. Because of the relatively long wavelength $(620 \mathrm{pm})$ compared to the known iodine-iodine distance (700 pm), the scattering extends to large angles and the first scattering maximum from the iodine-iodine interference is not covered by the detector in our setup. The experimentally obtained $I(s)$ is best fitted for an iodine-iodine distance of

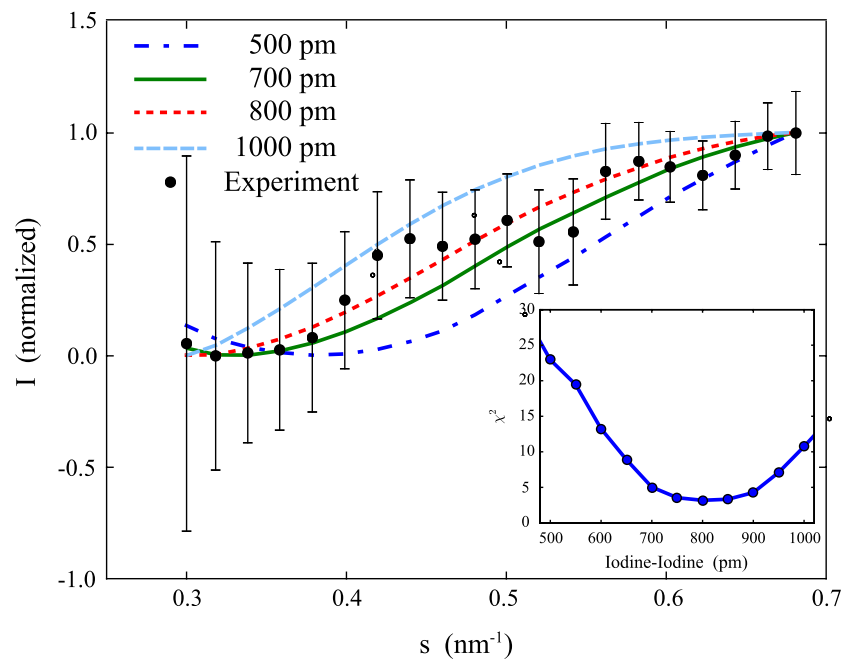

FIG. 4 (color online). Comparison of experimentally obtained intensity profiles $I(s)$ along the alignment direction of the diffraction-difference pattern $\mathrm{I}_{\mathrm{YAG}}-\mathrm{I}_{\text {NOYAG }}$ with simulated profiles. The experimentally obtained $I(s)$ is best fitted (in terms of a $\chi^{2}$ test) with the model for an iodine-iodine distance of $800 \mathrm{pm}$ (inset: test-statistic $\chi^{2}$ in dependence of the iodine-iodine distance).
$800 \mathrm{pm}$. Figure 4 shows the simulated $I(s)$ for iodine-iodine distances of 500, 700, 800, and 1000 pm. The inset of Fig. 4 depicts the calculated $\chi^{2}$ values [44] in dependence of the iodine-iodine distance. Because of the experimental parameters, as mentioned above, the scattering features are large and vary only slightly within the recorded range of $s$ values. We note that the structural features of small molecules, like DIBN, could be determined much more accurately with data recorded at a shorter wavelength where the available $s$ range extends to cover several maxima and minima. This would be possible at wavelengths of $200-100 \mathrm{pm}$, which became available at LCLS recently and will be available at upcoming facilities, e.g., the European XFEL, in the near future.

We do not observe direct signs of radiation damage in the diffraction data. While previous experiments aimed specifically at the investigation of $\mathrm{x}$-ray induced damage in strongly focused $\mathrm{x}$-ray beams $[8,45]$, here, we have actively avoided that regime and performed the experiments using a hundred times larger cross section of the x-ray beam. Under these moderate-fluence conditions the damage can be rationalized based on simple cross-section estimates for photoionization and elastic scattering and is detailed in the Supplemental Material [41]. Since the sample is replenished for every XFEL pulse, the diffractive imaging signal is only sensitive to the dynamics of damaged molecules during the $\mathrm{x}$-ray pulse ( $\sim 100 \mathrm{fs})$. Using a simple mechanical model, we estimate that most ( $90 \%)$ of the diffraction signal is due to (practically) intact molecules. A minor fraction of the signal is due to damaged molecules with small changes in molecular structure, which could not have been resolved with the available x-ray wavelength. Damage could even be mitigated using shorter ( $10 \mathrm{fs})$ duration pulses; see Supplemental Material [41] for details. Moreover, an appropriate trade-off between pulse duration, pulse energy, and repetition rate would allow the recording of atomically resolved $\mathrm{x}$-ray diffraction patterns of molecules within minutes [13]. At these high repetition rates, one could directly observe femtosecond molecular dynamics through snapshots for many time delays in pump-probe experiments of electronic-ground-state chemical dynamics.

In summary, we demonstrate the preparation of strongly aligned samples of polyatomic molecules at an XFEL facility. We experimentally verify that the high-frequency, far off-resonant $\mathrm{x}$ rays are an ideal probe of alignment of molecular ensembles in a photoion momentum imaging approach. The employed setup and conditions are applicable for coherent diffractive imaging of single biomolecules or molecular ensembles. We show the possibility to perform spatially resolved single $\mathrm{x}$-ray photon counting. Because of the weak scattering signal from small isolated molecules, averaging of many shots is necessary and possible for the observation of an analyzable diffraction signal, on top of a large background from NIR photons. We confirm that the angular structures in the single molecule diffraction patterns were preserved during averaging and 
that a diffraction pattern of isolated and strongly aligned DIBN molecules was successfully measured beyond experimental noise. Even with the experimentally limited range of scattering vectors $s$, the heavy-atom distance derived from the $I(s)$ plot is in agreement with the computed molecular structure, demonstrating the capability to extract structural information for small molecules.

Our results provide direct evidence for the feasibility of $\mathrm{x}$-ray diffractive imaging of aligned gas-phase ensembles of molecules. Analyzing radiation damage in detail shows that damage effects in the diffraction pattern could be avoided by using shorter $\mathrm{x}$-ray pulses with lower fluences at higher repetition rates. This would allow us to observe atomically resolved snapshots of ultrafast chemical dynamics. Combined with advanced molecular beam delivery techniques, e.g., laser desorption or helium droplet beams, considerably larger molecules could be delivered in cold beams, isomer selected, and aligned, providing a bottom-up approach toward the envisioned atomic-resolution singlemolecule diffraction experiments. In contrast to ultrafast electron diffraction, pump-probe experiments with $\mathrm{x}$-ray pulses will not suffer from Coulomb-repulsion broadening or pump-probe velocity mismatch and, hence, may permit better time resolution, i.e., in the range of 10-100 fs.

Parts of this research were carried out at the Linac Coherent Light Source (LCLS) at the SLAC National Accelerator Laboratory. LCLS is an Office of Science User Facility operated for the U. S. Department of Energy Office of Science by Stanford University. We acknowledge the Max Planck Society for funding the development and operation of the CAMP instrument within the ASG at CFEL. H.S. acknowledges support from the Carlsberg Foundation. C. C. and P. J. acknowledge support from the Swedish Research Council and the Swedish Foundation for Strategic Research. J.C.H.S. and H.N.C. acknowledge NSF STC Grant No. 1231306. A. Ru. acknowledges support from the Chemical Sciences, Geosciences, and Biosciences Division, Office of Basic Energy Sciences, Office of Science, U.S. Department of Energy. D. R. acknowledges support from the Helmholtz Gemeinschaft through the Young Investigator Program. This work has been supported by the excellence cluster "The Hamburg Center for Ultrafast Imaging-Structure, Dynamics and Control of Matter at the Atomic Scale" of the Deutsche Forschungsgemeinschaft.

*jochen.kuepper@cfel.de; http://desy.cfel.de/cid/cmi ${ }^{\mathrm{a}}$ Present address: Bruker AXS GmbH, Karlsruhe, Germany ${ }^{b}$ Present address: ARC Centre of Excellence for Coherent X-ray Science, School of Physics, The University of Melbourne, Australia

${ }^{c}$ Present address: Deutsches Elektronen-Synchrotron (DESY), 22607 Hamburg, Germany
${ }^{\mathrm{d}}$ Present address: Physikalisch-Technische Bundesanstalt, Bundesallee 100, 38116 Braunschweig, Germany

${ }^{e}$ Present address: European X-Ray Free Electron Laser (XFEL) GmbH, 22761 Hamburg, Germany

${ }^{\mathrm{f}}$ Stanford PULSE Institute, SLAC National Accelerator Laboratory, 2575 Sand Hill Road, Menlo Park, California 94025, USA

${ }^{g}$ Present address: PNSensor GmbH, 81739 Munich, Germany

${ }^{\mathrm{h}}$ Present address: Institute for Molecules and Materials, Radboud University Nijmegen, Heijendaalseweg 135, 6525 AJ Nijmegen, Netherlands

[1] R. Neutze, R. Wouts, D. van der Spoel, E. Weckert, and J. Hajdu, Nature (London) 406, 752 (2000).

[2] J. Andruszkow et al., Phys. Rev. Lett. 85, 3825 (2000).

[3] H. N. Chapman et al., Nat. Phys. 2, 839 (2006).

[4] A. Barty et al., Nat. Photonics 2, 415 (2008).

[5] Y. H. Jiang et al., Phys. Rev. Lett. 105, 263002 (2010).

[6] P. Emma et al., Nat. Photonics 4, 641 (2010).

[7] C. Bostedt et al., J. Phys. B 46, 164003 (2013).

[8] L. Young et al., Nature (London) 466, 56 (2010).

[9] H. N. Chapman et al., Nature (London) 470, 73 (2011).

[10] M. Marvin Seibert et al., Nature (London) 470, 78 (2011).

[11] J. M. Glownia et al., Opt. Express 18, 17620 (2010).

[12] R. Boll et al., Phys. Rev. A 88, 061402 (2013).

[13] A. Barty, J. Küpper, and H. N. Chapman, Annu. Rev. Phys. Chem. 64, 415 (2013).

[14] N. D. Loh et al., Nature (London) 486, 513 (2012).

[15] T. Gorkhover et al., Phys. Rev. Lett. 108, 245005 (2012).

[16] A. Barty et al., Nat. Photonics 6, 35 (2012).

[17] F. Filsinger, G. Meijer, H. Stapelfeldt, H. Chapman, and J. Küpper, Phys. Chem. Chem. Phys. 13, 2076 (2011).

[18] G. von Helden, T. Wyttenbach, and M. T. Bowers, Science 267, 1483 (1995).

[19] S. Trippel, Y.-P. Chang, S. Stern, T. Mullins, L. Holmegaard, and J. Küpper Phys. Rev. A 86, 033202 (2012).

[20] F. Filsinger, J. Küpper, G. Meijer, J. L. Hansen, J. Maurer, J. H. Nielsen, L. Holmegaard, and H. Stapelfeldt, Angew. Chem. Int. Ed. 48, 6900 (2009).

[21] T. Kierspel, D. A. Horke, Y.-P. Chang, and J. Küpper, Chem. Phys. Lett. 591, 130 (2014).

[22] F. Filsinger, U. Erlekam, G. von Helden, J. Küpper, and G. Meijer, Phys. Rev. Lett. 100, 133003 (2008).

[23] H. Stapelfeldt and T. Seideman, Rev. Mod. Phys. 75, 543 (2003).

[24] J. C. H. Spence and R. B. Doak, Phys. Rev. Lett. 92, 198102 (2004).

[25] J. C. H. Spence, K. Schmidt, J. S. Wu, G. Hembree, U. Weierstall, R. B. Doak, and P. Fromme, Acta Crystallogr. Sect. A 61, 237 (2005).

[26] E. R. Peterson et al., Appl. Phys. Lett. 92, 094106 (2008).

[27] S. Pabst, P. J. Ho, and R. Santra, Phys. Rev. A 81, 043425 (2010).

[28] C. J. Hensley, J. Yang, and M. Centurion, Phys. Rev. Lett. 109, 133202 (2012).

[29] U. Lorenz, N. M. Kabachnik, E. Weckert, and I. A. Vartanyants, Phys. Rev. E 86, 051911 (2012).

[30] B. Ziaja, H. N. Chapman, R. Fäustlin, S. Hau-Riege, Z. Jurek, A. V. Martin, S. Toleikis, F. Wang, E. Weckert, and R. Santra, New J. Phys. 14, 115015 (2012). 
[31] A. Fratalocchi and G. Ruocco, Phys. Rev. Lett. 106, 105504 (2011).

[32] L. Holmegaard, J. H. Nielsen, I. Nevo, H. Stapelfeldt, F. Filsinger, J. Küpper, and G. Meijer, Phys. Rev. Lett. 102, 023001 (2009).

[33] J. J. Larsen, K. Hald, N. Bjerre, H. Stapelfeldt, and T. Seideman, Phys. Rev. Lett. 85, 2470 (2000).

[34] I. Nevo, L. Holmegaard, J. H. Nielsen, J. L. Hansen, H. Stapelfeldt, F. Filsinger, G. Meijer, and J. Küpper, Phys. Chem. Chem. Phys. 11, 9912 (2009).

[35] J. L. Hansen, J. J. Omiste Romero, J. Hedegaard Nielsen, D. Pentlehner, J. Küpper, R. González-Férez, and $\mathrm{H}$. Stapelfeldt, J. Chem. Phys. 139, 234313 (2013).

[36] L Strüder et al., Nucl. Instrum. Methods Phys. Res., Sect. A 614, 483 (2010).
[37] L. Foucar et al., Comput. Phys. Commun. 183, 2207 (2012).

[38] F. Filsinger, J. Küpper, G. Meijer, L. Holmegaard, J. H. Nielsen, I. Nevo, J. L. Hansen, and H. Stapelfeldt, J. Chem. Phys. 131, 064309 (2009).

[39] U. Even, J. Jortner, D. Noy, N. Lavie, and N. CossartMagos, J. Chem. Phys. 112, 8068 (2000).

[40] B. Rudek et al., Nat. Photonics 6, 858 (2012).

[41] See Supplemental Material at http://link.aps.org/ supplemental/10.1103/PhysRevLett.112.083002 for details.

[42] D. Waasmaier and A. Kirfel, Acta Crystallogr. Sect. A 51, 416 (1995).

[43] M. W. Schmidt et al., J. Comput. Chem. 14, 1347 (1993).

[44] K Nakamura (Particle Data Group), J. Phys. G 37, 075021 (2010), Section 33 (Statistics).

[45] B. Erk et al., Phys. Rev. Lett. 110, 053003 (2013). 